\title{
Reversal of Antibiotic Resistance by Phage Resistant Pseudomonas aeruginosa PA01
}

\section{Running Title: Interaction of Multiple Drug Resistant Host Biofilm with Potent Ghost}

\author{
Sadhana Singh Sagar,", Ram Narain ${ }^{2}$, Rajesh Kumar ${ }^{3}$, Shilpa Deshpande Kaistha ${ }^{4}$ \\ ${ }^{1}$ Department of Microbiology, Rani Lakshmi Bai Central Agricultural University, Jhansi, 284003, India \\ ${ }^{2}$ Department of Biotechnology, Veer Bahadur Singh Purvanchal University, Jaunpur, India \\ ${ }^{3}$ Department of Environmental Microbiology, Babasaheb Bhimrao Ambedkar University (A Central University), \\ Lucknow-226025, India \\ ${ }^{4}$ Department of Microbiology, Institute of Bioscience and Biotechnology, Chhatrapati Sahu Ji Maharaj University, \\ Kanpur -208024, India
}

Copyright $(2018$ by authors, all rights reserved. Authors agree that this article remains permanently open access under the terms of the Creative Commons Attribution License 4.0 International License

\begin{abstract}
Emergence of antibiotic resistance is a serious cause of concern worldwide. Limitation in the discovery of new antibiotics and emergence of resistant infectious microorganisms has directed the attention of researcher toward the phage therapy. Phages are obligate parasite of bacteria and they are highly specific for their host. Phage therapy is an ideal strategy for the control of such infectious pathogens. Pseudomonas aeruginosa PA01 is multiple drug resistant (MDR) bacteria, biofilm forming nosocomial pathogen. Phage therapy of PA01 was studied using bacteriophage $\varphi s 1$ isolated from Ganga water, India. During this study, we observed PA01 variants that had evolved resistant mechanism against phage $\varphi s 1$ infection. These phage resistance cells showed variation in their pigment production from green to brown and also showed reversion of antibiotic resistance forerythromycin (ERT), norfloxacin (NRF), kanamycin (KAN), gentamicin (GEN), gatifloxacin (GAT), and imipenem (IMP) class of antibiotics. Biofilm forming ability of prPA01 also decreased by up to $90 \%$ and they became more susceptible for imipenem antibiotic as compare to PA01.
\end{abstract}

Keywords Pseudomonas aeruginosa, Biofilm, Imipenem, Bacteriophage and TEM

\section{Introduction}

Despite modern antibiotics, infectious diseases are still associated with health-problems and are responsible for the deaths worldwide (Schmidt et al., 2008) ${ }^{[1]}$. Multiple drug resistant bacteria have evolved resistant mechanism against almost all available antibiotics. Pseudomonas aeruginosa is one of most common opportunistic pathogens associated with nosocomial infection. $P$. aeruginosa have evolved a variety of resistance mechanisms against different classes of antibiotics, particularly carbapenems. Meropenem and imipenem are most widely used antibiotics against gram positive and gram negative bacteria (Shahid et al., 2009) ${ }^{[2]}$. According to European Centre for Disease prevention and control, $18 \%$ P. aeruginosa have evolved resistance mechanism against the carbapenems ${ }^{[3]}$. It was suggested that the carbapenems are the last line of drug for the gram negative bacteria (Falagas and Karageorgopoulos, $2008)^{[4]}$. But unfortunately, bacteria have evolved resistant mechanism against carbapenems and P. aeruginosa is one of them. Lautenbach et al. ${ }^{[5]}$, reported that patient with imipenem resistant $P$. aeruginosa infection show high mortality rates. Phage therapy has brought new hope to fight against such last drug resistant bacteria. Bacteriophages are obligate parasite of bacteria. Application of bacteriophage for the control of MDR pathogens is an ecofriendly and economical approach. However, a major drawback of phage therapy also is the rapid development of phage resistance by host pathogen. Basically, bacteria become resistant against phages by, restriction the entry of phages, adsorption blocking and by CRISPR mechanism (Labrie et al., 2010 and Sagar et al., 2017) ${ }^{[6,7]}$.

In the described system, phages resistant host cell reverse the pathogen's drug resistance, thereby restoring their sensitivity to antibiotics. A drug sensitizing DNA cassette was transferred into using lysogenization by phages rendering previously resistant pathogens to 
sensitive variants (Lederberg, 1951) ${ }^{[8]}$.

In the current study, phage resistant PA01 (prPA01) were isolated in biocontrol studies with isolated bacteriophage $\phi s 1$. We isolated those phage resistant cells and analyzed them for the antibiotic susceptibility and its virulence traits. The results were very surprising, prPA01 reverse their resistant for the erythromycin (ERT), norfloxacin (NRF), kanamycin (KAN), gentamicin (GEN), gatifloxacin (GAT), and imipenem (IMP) antibiotics and its virulent traits were suppressed as well. Colony characteristics were also changed from green to brown with wrinkled colony characteristics.

\section{Materials and Methods}

\section{Bacterial Strain and Growth Media}

Standard strain $P$. aeruginosa PA01 was used as host for bacteriophage isolation studied. The bacteria were maintained on Tryptone Soya Broth (TSB) medium at $37^{\circ} \mathrm{C}$.

\section{Isolation of Bacteriophages}

The enrichment method as per Cerveny et al. ${ }^{[9]}$ wasadopted for the isolation of phage specific to $P$. aeruginosa PA01 from Ganges river at the Kanpur city, India. These sites were selected to isolate phages, as sewage water is known to harbor many different bacteria and hence the likelihood of prevalence of phages against different microorganisms. Briefly, sewage samples were collected; centrifuged $\left(10,000 \mathrm{rpm}, 10 \mathrm{~min}, 4^{\circ} \mathrm{C}\right)$ and supernatants were filter sterilized $(0.45 \mu \mathrm{m}$ pore size Millipore filter). $5 \mathrm{ml}$ filtered sewage sample and $5 \mathrm{ml}$ sterile SM buffer were mixed with $5.0 \mathrm{ml}$ overnight culture of $P$. aeruginosa PA01 and incubated at $37^{\circ} \mathrm{C}$ overnight. The bacteria were removed by centrifugation; supernatant was filtering sterilized and checked for the presence of phages.

\section{Biofilm Formation Assay \& Hemolytic Assay}

The ability of PA01 to form biofilms was determined using the crystal violet binding assay as well as Congo Red Assay (CRA). Briefly, isolate was streaked on Congo Red Agar and the presence of black precipitate of Congo Red on colonies after $24 \mathrm{~h}$ incubation at $37^{\circ} \mathrm{C}$ was indicative of biofilm formation. In the crystal violet binding assay, log phage isolates were cultured in microtiter plates with different treatments in TSB for $24 \mathrm{~h}$ at $37^{\circ} \mathrm{C}$. Post incubation, ability of isolate to form biofilm on microtiter surface was assayed by staining with crystal violet and absorbance measured at $540 \mathrm{~nm}$. Hemolytic ability of the isolate was determined by streaking on blood agar plate and observing hemolytic zone following incubation.

\section{Spot Test and Plaque Assay}

To detect the presence of phages in supernatant, spot test was carried out as described by Chang et al. ${ }^{[10]}$. The phage titer was determined by plaque assay by employing double agar overlay technique. Briefly, each of the phage suspension was serially diluted. $10 \mu$ l diluted phage were spot inoculated on molten agar $(0.8 \%$ agar, $w / v)$ containing host cells of $10^{7} \mathrm{CFU} / \mathrm{ml}$ as per Chang et al. ${ }^{[10]}$. Clear zone of plaques were observed after incubating the plates overnight at $37^{\circ} \mathrm{C}$.

\section{Electron Microscopy}

To observe phage morphology, transmission electron microscopy of $P$. aeruginosa PA01 specific phages was performed as described by Goodridge et al. ${ }^{[11]}$ with some modification. Drops of ultracentrifuge phage samples were dropped on copper coated grids (diameter, $3 \mathrm{~mm}$; 300 meshes). After $5 \mathrm{~min}$, the phage particles were stained with $2 \%(\mathrm{w} / \mathrm{v})$ phosphotungstic acid (PTA) for $10 \mathrm{~s}$. The grids were allowed to dry for $20 \mathrm{~min}$ and examined under a transmission electron microscope (FEI Tecnai S Twin) at $200 \mathrm{Kv}$.

\section{MIC Test of PA01 and prPA01 with Imipenem Antibiotic}

Culture of $P$. aeruginosa PA01 and prPA01 were grown in TSB (OD560 of 0.1) having cell count of $10^{8} \mathrm{cfu} / \mathrm{ml}$ The culture $(190 \mu \mathrm{l})$ was dispensed in 96 wells of sterile polystyrene microtiter plate with imipenem antibiotic with various concentrations $(0.1,0.075,0.03$, $0.01,0.005,0.002,0.0005$, and $0.0002 \mathrm{mg} / \mathrm{ml})$. Culture was incubated for the $24 \mathrm{~h}$ at $37^{\circ} \mathrm{C}$. After incubation MTT assay was performed as per Abate et al., ${ }^{[12]}$ with some modification.

For the biofilm formation assay, biofilm cells well incubated with $0.3 \%$ MTT dye solution in PBS for $30 \mathrm{~min}$ under dark condition. After incubation, stained cells were dissolved in DMSO and absorbance was taken at A540.

\section{Results and Discussion}

\section{Selection of Phage Resistant $P$. aeruginosa:}

Bacteriophage $\varphi s 1$ was isolated from the Ganga river water using PA01 as host bacterium. The lytic phage was characterized for its ability to lyse host PA01 and within the plaque isolated, a number of resistant cells were observed (Figure 1A). These phage resistant variant were isolated on TSA and characterized as prPA01 (Figure 1B). Interestingly, the phage resistant prPA01 showed change in pigment production from green to dark brown. Similar 
report has previously been published by $\mathrm{Li}$ et al., ${ }^{[13]}$ wherein chromosomal DNA alteration rendered a Pseudomonas resistant to phage. They reported that red color pigment production in $P$. aeruginosa PA1r is due to deletion of gene hmgA on phage infection, apart from this galU is placed in front hmgA which impart resistance to PA1r against phage by mutation of LPS receptor. The transmission electron micrograph of phage $\phi s 1$ shows that it's a tailed icosahedral head phage belonging to Myoviridae (Figure 1C).

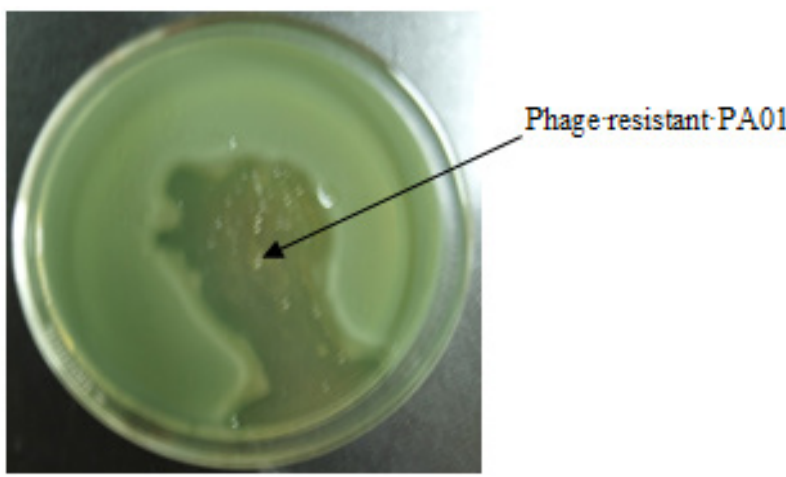

Figure 1A. Development of phage resistant prPA01 isolates in spot assay with bacteriophage $\phi s 1$

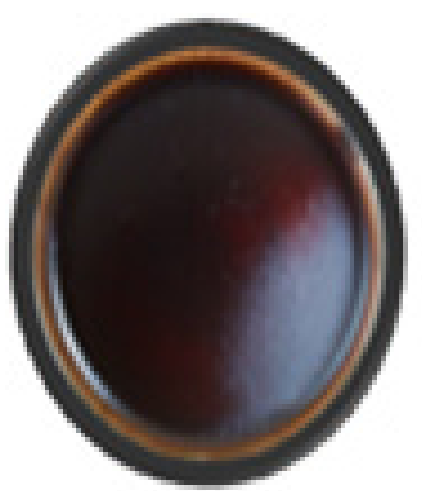

Figure 1B. Change in colony morphology of prPA01 from green to brown pigment

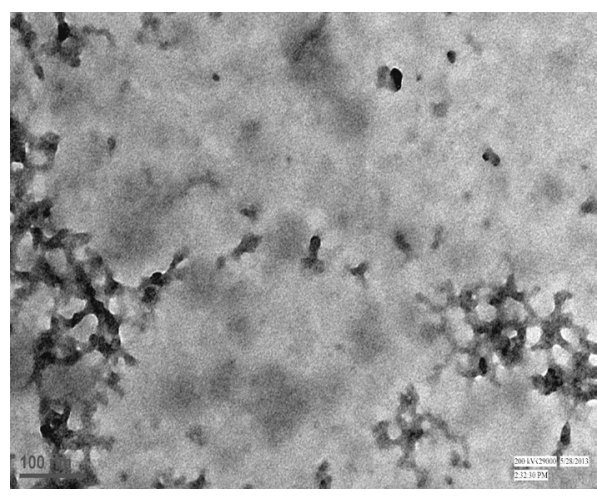

Figure 1C. Electron Micrograph of phage $\phi s 1$

\section{Characteristics of P. aeruginosa PA01 and Phage Resistant (prPA01)}

Comparison of the host PA01 and the phage resistant prPA01 was further performed based on antibiotic sensitivity and their ability to form biofilms. Antibiotic resistance profile of $P$. aeruginosa PA01 was determined using the Kirby Bauer disk diffusion assay as per CLSI norms. Antibiotic susceptibility test was performed with Erythromycin (ERT), Norfloxacin (NRF), Penicillin (PEN), Amikacin (AMK), Kanamycin (KAN), Gentamicin (GEN), Gatifloxacin (GAT), Imipenem (IMP). On the basis of antibiotic susceptibility test it was confirmed that PA01 was highly resistant for the used antibiotics (Table-1). Furthermore, biofilm forming ability and pathogenicity of PA01 was determined by CV and congo red binding assay and $\beta$ hemolytic activity respectively. $\mathrm{CV}$ assay was done to determine for the quantitative analysis of biofilm forming ability of PA01, while congo red binding assay was done for the qualitative analysis for biofilm forming ability of PA01. Table 1 and Figure 2A show that the phage resistant variant prPA01 also showed variation in its phenotypic display of antibiotic susceptibility to ERT, NRF, KAN, GEN, GAT and IMP antibiotics. prPA01 had also lost its ability for biofilm formation as determined by two independent assays.

Table 1. Characteristics of $P$. aeruginosa PA01 and phage resistant PA01 (prPA01):

\begin{tabular}{cccccccccccc}
\hline \multirow{2}{*}{$\begin{array}{c}\text { Strain } \\
\text { name }\end{array}$} & \multicolumn{1}{c}{ Antibiotic resistance profile of strains } & \multicolumn{4}{c}{ Biofilm forming ability } & \multicolumn{2}{c}{$\begin{array}{c}\beta \text {-hemolytic } \\
\text { activity }\end{array}$} \\
\cline { 2 - 14 } & ERT & NRF & PEN & AMK & KAN & GEN & GAT & IMP & CV assay & $\begin{array}{c}\text { Congo Red } \\
\text { assay }\end{array}$ \\
\hline PA01 & R & R & R & R & R & R & R & R & $1.61933 \pm 0.0381$ & +++ & ++ \\
\hline prPA01 & S & S & R & R & S & S & S & S & $0.47033 \pm 0.0472$ & - & ND \\
\hline
\end{tabular}

Antibiotic resistance profile was determined according to CLSI guideline; ND-not done; CV-Crystal Violet assay (provided data is in the form of absorbance average \pm standard deviation (value above0.6 is high biofilm former, while below 0.6 is poor biofilm former) 
In order to determine the extent of change in antibiotic sensitivity, minimum inhibitory concentration (MIC) test for the imipenem was conducted with prPA01 cells. MIC test was done with various concentrations of imipenem antibiotics $(0.1,0.075,0.03,0.01,0.005,0.002,0.0005$, and $0.0002 \mathrm{mg} / \mathrm{ml}$ ) against PA01 and prPA01. MIC data revealed that PA01 biofilm forming ability was slightly affected with 0.1 and $0.075 \mathrm{mg} / \mathrm{ml}$, while on decreasing concentration of antibiotic, biofilm cells survival rate increased (Figure.3B). In case of prPA01, 0.1, 0.075, 0.03, and $0.01 \mathrm{mg} / \mathrm{ml}$ concentration of imipenem antibiotic caused killing of biofilm cells by up to $90 \%$ (Figure 3B). MTT dye was used to determine the strength of live and dead biofilm cell (Figure 3C). MIC data determined that prPA01 reduced their biofilm forming by many fold as compared to PA01. The present study revealed that phage resistant cells sensitizes for the antibiotic imipenem and other used antibiotics. Chan et al. ${ }^{[14]}$ have explained in their study that genetic trade offare often observed in the field of biology where at one side organism evolved a trade for their fitness and simultaneously suffering in performance in another trait. Edgar et al., ${ }^{[15]}$, reported in their study that lysogenic phage M13 sensitized E. coli for the nalidixic acid and streptomycin by disrupting the SOS gene response Edgar et al., ${ }^{[15]}$. This finding can bring a hope for the medical practitioners when bacteria have evolved resistance against the almost all antibiotics and bacteriophages as well.

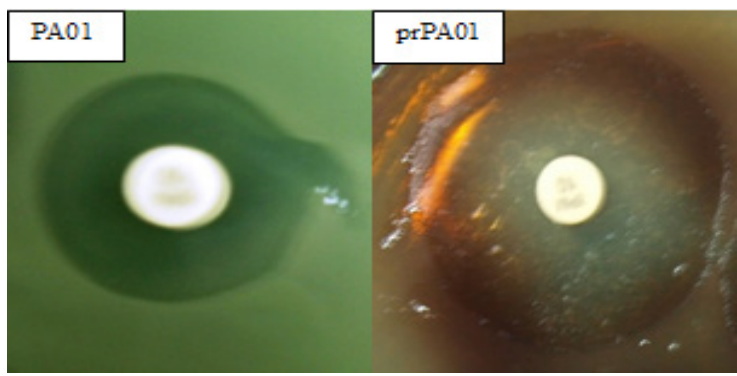

Figure 2A. Picture of antibiotic susceptibility test of PA01 and prPA01

\section{MIC of Imipenem for biofilm of phage resistant cells}

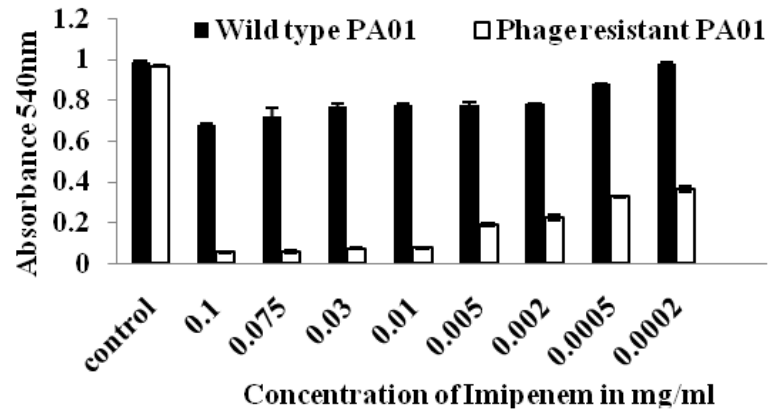

Figure 3B. MIC of Imipenem for biofilm of phage resistant cells

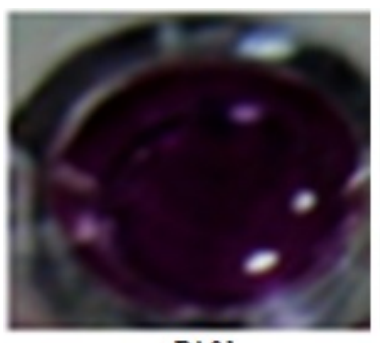

PA01

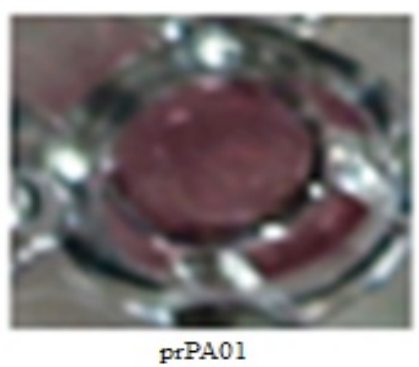

prPA01
Figure 3C. Picture of biofilm of Phage resistant cells on micro titer plate stained with MTT dye:

\section{Conclusions}

The MDR bacteria are spreading their claws for capturing new hosts and have left little option for the medical practitioners. Medical societies have high hope with the phage therapy but unfortunately, bacteria have evolved a variety of resistant mechanism against them. Hence, currently we don't have any option for controlling of such superbugs. Fortunately, it was observed that phage resistant cells reverses their traits such as resistance to antibiotics and become less virulent. This is a novel finding and can bring a new hope for the medical society by understand the mechanism of phage resistant cells.

\section{REFERENCES}

[1] Schmidt, I., Aigner and Schavan, A., (2008). DART -Deutsche Antibiotika Resistens Strategie. Bundesministerium fuer Gesundhe it, November, 13

[2] Shahid, M., Sobia, F., Singh, A. et al. (2009). Beta-lactams and beta-lactamase inhibitors in current-or potential-clinical practice: A comprehensive update. Critical Review in Microbiology 35:81-108.

[3] European Centre for Disease Prevention and Control. Antimicrobial resistance surveillance in Europe (2013). Annual Report of the European Antimicrobial Resistance Surveillance Network (EARS-Net), 1-2

[4] Falagas, M.E., and Karageorgopoulos, D.E. (2008). Pandrug resistance (PDR), extensive drug resistance (XDR), and multidrug resistance (MDR) among Gram-negative bacilli: need for international harmonization in terminology. Clinical Infectious Diseases 46: 1121-1122.

[5] Lautenbach, E., Synnestvedt, M., Weiner, M.G., Bilker, W.B., Vo, L., Schein, J., Kim, M. (2010). Imipenem Resistance in Pseudomonas aeruginosa Emergence, Epidemiology, and Impact on Clinical and Economic Outcomes. Infection Control \& Hospital Epidemiology, 31(1):47-53.

[6] Labrie, S.J., Samson, J.E. \& Moineau, S. (2010). Bacteriophage resistance mechanisms. Nature Review in 
Microbiology 8:317-327.

[7] Sagar, S.S., Kumar, R., Kaistha, S.D. (2017). Efficacy of Phage and Ciprofloxacin Co-therapy on the Formation and Eradication of Pseudomonas aeruginosa Biofilms. Arabian Journal for Science and Engineering 42(1):95-103.

[8] Lederberg, J. (1951). Streptomycin resistance; a genetically recessive mutation. Journal of Bacteriology, $61: 549-550$

[9] Cerveny, K.E., DePaola, A., Duckworth, D.H. \& Gulig, P.A. (2002). Phage therapy of local and systemic disease caused by Vibrio vulnificus in iron- dextran -treated mice. Infection and Immunity, 70(11): 6251-6262.

[10] Chang, H.C., Chen, C.R., Lin, J.W., Shen, G.H., Chang, K.M., Tseng, Y.H. \& Weng, S.F. (2005). Isolation and characterization of novel giant Stenotrophomonas maltophilia phage ФSMA5. Applied and Environmental Microbiology 71:1387-1393

[11] Goodridge, L., Gallacci, A., Griffiths, M.W. (2003). Morphological, host range and genetic characterization of two coliphages. Applied Environmental Microbiology, 69(9), 5364-5371.

[12] Abate, G., Mshana, R.N., Miorner, H. (1998). Evaluation of a colorimetric assay based on 3-(4,5-dimethylthiazol-2-yl)-2,5-diphenyl tetrazolium bromide (MTT) for rapid detection of rifampicin resistance in Mycobacterium tuberculosis. International Journal of Tuberculosis Lung Diseases 2:1011-1016.

[13] Le, S., Yao, X., Lu, S., Tan, Y., Rao, X., Li, M., Jin, X., Wang, J., Zhao, Y., Wu, N.C., Lux, R. (2014). Chromosomal DNA deletion confers phage resistance to Pseudomonas aeruginosa. Scientific reports, 4.

[14] Chan, B.K., Sistrom, M., Wertz, J.E., Kortright, K.E., Narayan, D. and Turner, P.E. (2016). Phage selection restores antibiotic sensitivity in MDR Pseudomonas aeruginosa. Scientific reports 6:26717.

[15] Edgar, R., Friedman, N., Molshanski-Mor, S., Qimron, U. (2012). Reversing bacterial resistance to antibiotics by phage-mediated delivery of dominant sensitive genes. Applied and environmental microbiology. 78(3):744-51. 